\title{
Fréedericksz-like positional transition
}

\author{
Ke Xiao, Xi Chen, and Chen-Xu Wu ${ }^{*}$ \\ Department of Physics, Jiujiang Research Institute, Xiamen University, Xiamen 361005, People's Republic of China
}

(Received 11 July 2019; published 22 October 2019)

\begin{abstract}
In this paper, a Fréedericksz-like positional transition is found for a spherical microdroplet suspended in a nematic liquid crystal cell in the presence of an external electric field. Based on the numerical calculation of elastic energy using the Green's function method, the equilibrium position of the microdroplet is decided through a competition between the buoyant force and the effective force built by the elastic energy gradient existing inside the nematic liquid crystal cell. It is shown that elastic energy dominates the kinetics of the microdroplet until the external field applied reaches a critical value large enough to flatten the elastic energy contour in the central region, which provides a "fast lane" enabling the asymmetric buoyant force to drive the liquid droplet abruptly from the cell midplane to a new equilibrium position, triggering a positional transition. It is also found that such a threshold value of external field depends on thickness $L$ and Frank elastic constant $K$, in a Fréedericksz-like manner, but multiplied by a factor of $3 \sqrt{\pi}$. An explicit formula proposed for the critical electric field agrees extremely well with the numerical calculation.
\end{abstract}

DOI: 10.1103/PhysRevResearch.1.033041

\section{INTRODUCTION}

Behaviors of immiscible liquid, solid, or gas microphase suspensions in a nematic liquid crystal (NLC) cell are of considerable interest due to their promising practical applications in new display devices and materials [1-3], triggered and released microcargo [4], and biological detectors [5,6]. So far much effort has been put forth to investigate the behaviors of microparticles suspended in confined NLCs by means of experiment and numerical modeling [7-16]. Physical phenomena such as levitation, lift, bidirectional motion, and aggregation of colloids induced by the effect of the electric field were also studied through experiments and computer simulations [17,18]. Diverse methods and techniques have been developed to measure the interaction force between particles in NLCs in a direct manner [7,19-22].

The interaction force of spherical particles suspended in NLC is associated not only with interparticle distance and geological confinement [8], but also with the shape of particles, which sometimes plays a crucial role in pair interaction and aggregation behaviors [12]. In most cases the inclusion of particles in a NLC cell tends to create liquid crystal (LC) alignment singularities around the suspended substances, which in general are determined by surface anchoring conditions, particle size, boundary conditions, and external fields [12,23-26]. It has been widely confirmed and accepted that when a spherical particle is immersed in a NLC, there exist two possible

\footnotetext{
*xwu@xmu.edu.cn

Published by the American Physical Society under the terms of the Creative Commons Attribution 4.0 International license. Further distribution of this work must maintain attribution to the author(s) and the published article's title, journal citation, and DOI.
}

types of defect configurations [27-30]. Dipole and quadrupolar configurations are usually seen around a spherical particle with strong vertical surface anchoring, whereas a boojum defect is formed by a microsphere with tangential surface anchoring. In addition, recently Senyuk et al. found that solid polymer microspheres dispersed in a NLC may induce surface point and line defects at the same time, and therefore lead to hexadecapolar distortions around the microspheres due to conically degenerate boundary condition [30]. Through experimental observations it has been found that when an external field is applied, there exists a transition between elastic dipole and quadrupolar configurations which depends on particle size and surface anchoring strength [31-33].

On the theoretical side, Chernyshuk et al. studied the interaction between colloidal particles in NLCs near one wall and in a nematic cell with or without an external field by using the Green's function method and obtained general formulas for the interaction energy between colloidal particles [34-36]. Although the interactions of two particles in a NLC are very well understood and the particle-wall interaction has been widely observed experimentally for a single particle immersed in a nematic cell $[35,37,38]$, the properties of a single particle in a uniform NLC cell in the presence of an external electric field theoretically have not been fully addressed.

\section{THEORETICAL MODELING}

The system we consider consists of a microdroplet of radius $r$ suspended in a NLC cell in the presence of an external electric field, as illustrated in Fig. 1. Experiments showed that typically such a liquid droplet tends to generate a companion hyperbolic hedgehog rather than a disclination ring [29], and thus it is appropriate and much more convenient to consider the microdroplet and its surrounding LC alignments as a dipole configuration. 


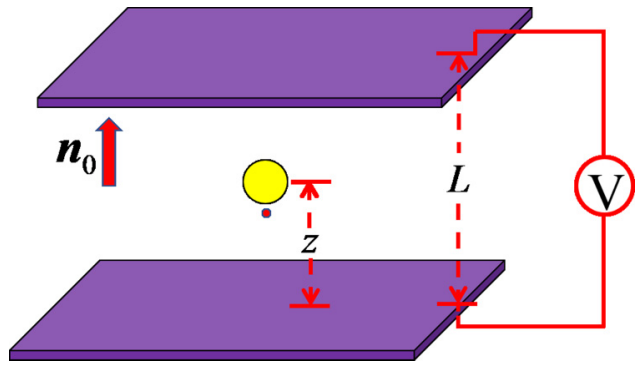

FIG. 1. Sketch of a microdroplet of radius $r$ suspended in a nematic cell with an $L$-thick spacer in the presence of an external field.

With one Frank constant approximation, the effective elastic energy for the system is given by [36]

$$
\begin{aligned}
U_{e}= & K \int d^{3} x\left[\frac{\left(\nabla n_{\mu}\right)^{2}}{2}-\frac{k^{2}}{2}(\mathbf{e n})^{2}-4 \pi P(\mathbf{x}) \partial_{\mu} n_{\mu}\right. \\
& \left.-4 \pi C(\mathbf{x}) \partial_{z} \partial_{\mu} n_{\mu}\right],
\end{aligned}
$$

where $K$ is the Frank constant, $n_{\mu}(\mu=x, y)$ represents the components of the director field $\mathbf{n}$ perpendicular to $\mathbf{n}_{0}$, and $k^{2}=(4 \pi K)^{-1} \Delta \varepsilon E^{2}$, with $\Delta \varepsilon=\varepsilon_{\|}-\varepsilon_{\perp}$ the dielectric anisotropy of the NLC, which is assumed to be positive in this paper. Here $P(\mathbf{x})$ and $C(\mathbf{x})$ denote the dipole- and the quadrupole-moment densities, respectively. For simplicity, we choose the coordinate $z$ axis along the normal direction of the two cell walls where LC molecules are homeotropically anchored. Given these, when an electric field along $z$ axis is applied, we have the Euler-Lagrange equations [36]

$$
\Delta n_{\mu}-k^{2} n_{\mu}=4 \pi\left[\partial_{\mu} P(\mathbf{x})-\partial_{z} \partial_{\mu} C(\mathbf{x})\right] .
$$

With Dirichlet boundary conditions $\left.n_{\mu}\right|_{(z=0)}=\left.n_{\mu}\right|_{(z=L)}=$ 0 for homeotropic anchoring on the two walls, the solution of the above equation can be written as [36]

$$
n_{\mu}(\mathbf{x})=\int_{V} d^{3} \mathbf{x}^{\prime} G_{\mu}\left(\mathbf{x}, \mathbf{x}^{\prime}\right)\left[-\partial_{\mu}^{\prime} P\left(\mathbf{x}^{\prime}\right)+\partial_{\mu}^{\prime} \partial_{z}^{\prime} C\left(\mathbf{x}^{\prime}\right)\right],
$$

where $G_{\mu}$ is the Green's function given by [36]

$$
\begin{aligned}
G_{\mu}\left(\mathbf{x}, \mathbf{x}^{\prime}\right)= & \frac{4}{L} \sum_{n=1}^{\infty} \sum_{m=-\infty}^{\infty} e^{i m\left(\varphi-\varphi^{\prime}\right)} \sin \frac{n \pi z}{L} \\
& \times \sin \frac{n \pi z^{\prime}}{L} I_{m}\left(\lambda_{n} \rho_{<}\right) K_{m}\left(\lambda n \rho_{>}\right) .
\end{aligned}
$$

Here $I_{m}$ and $K_{m}$ are modified Bessel functions and $\lambda_{n}=$ $\left[(n \pi / L)^{2}+\Delta \varepsilon E^{2} / 4 \pi K\right]^{1 / 2}$. Then substituting $H_{\mu}\left(\mathbf{x}, \mathbf{x}^{\prime}\right)=$ $G_{\mu}\left(\mathbf{x}, \mathbf{x}^{\prime}\right)-1 /\left|\mathbf{x}-\mathbf{x}^{\prime}\right|$ into the self-energy defined in terms of the Green's function in Ref. [36], we can obtain the elastic energy $U_{e}$ for a NLC cell with a microdroplet suspended in the presence of an electric field. Besides the elastic energy, the gravitational potential $U_{g}$ due to buoyant force should be considered as well, leading to a total energy written as

$$
\begin{aligned}
U_{\text {total }}= & U_{e}+U_{g} \\
= & -2 \pi K p^{2}\left[-\frac{4}{L} \sum_{n=1}^{\infty} \lambda_{n}^{2} \sin ^{2}\left(\frac{n \pi z}{L}\right) K_{0}\left(\lambda_{n} \rho\right)+\frac{1}{\rho^{3}}\right]_{\rho \rightarrow 0} \\
& -\frac{4}{3} \pi r^{3}\left(\rho_{\mathrm{LC}}-\rho_{a q}\right) g z
\end{aligned}
$$
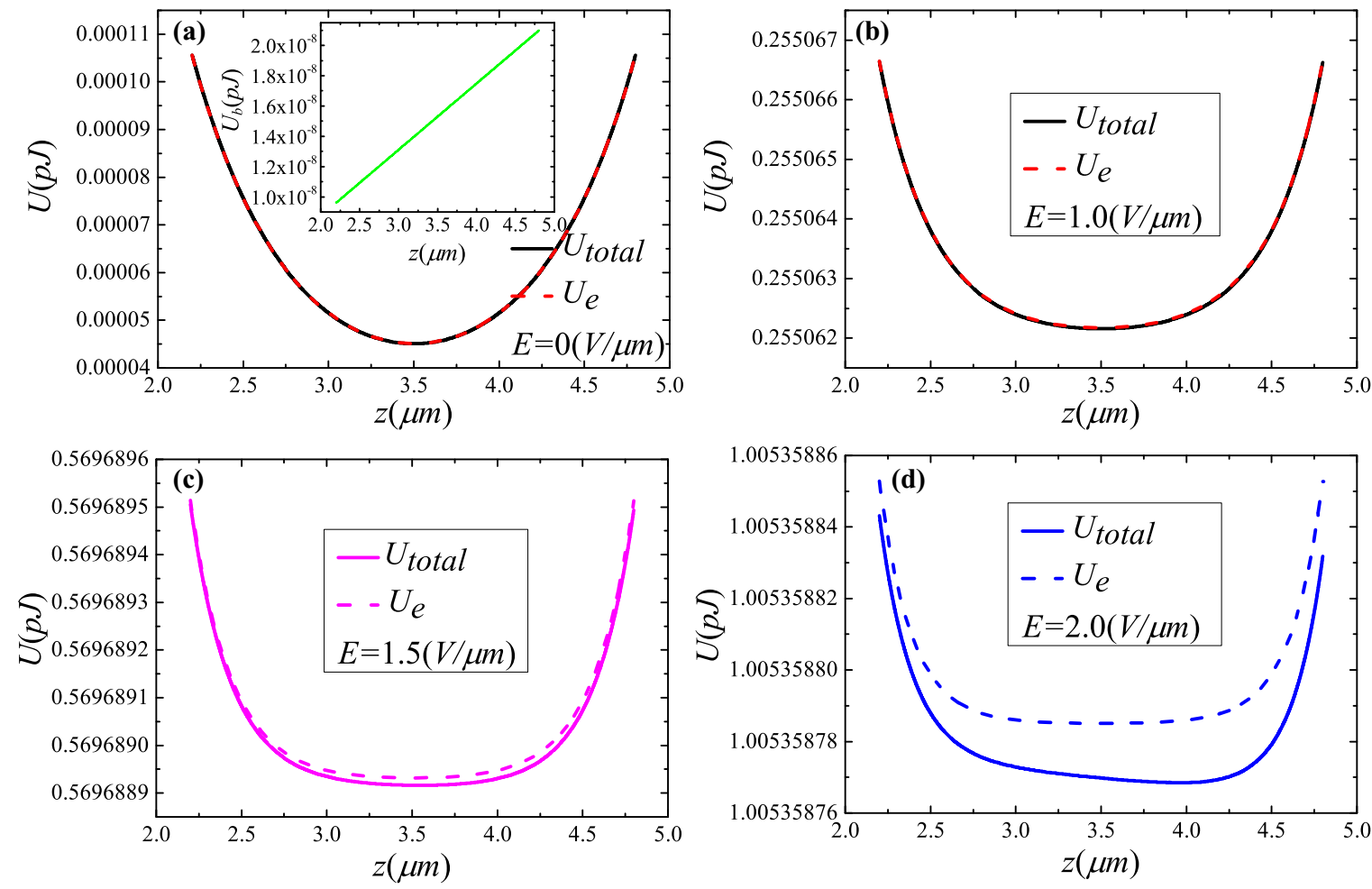

FIG. 2. Elastic energy and total energy as a function of microdroplet position for different electric fields $(0,1.0,1.5$, and $2.0 \mathrm{~V} / \mu \mathrm{m})$. Here we set the radius of the microdroplet and cell thickness as 2.2 and $7 \mu \mathrm{m}$, respectively. 

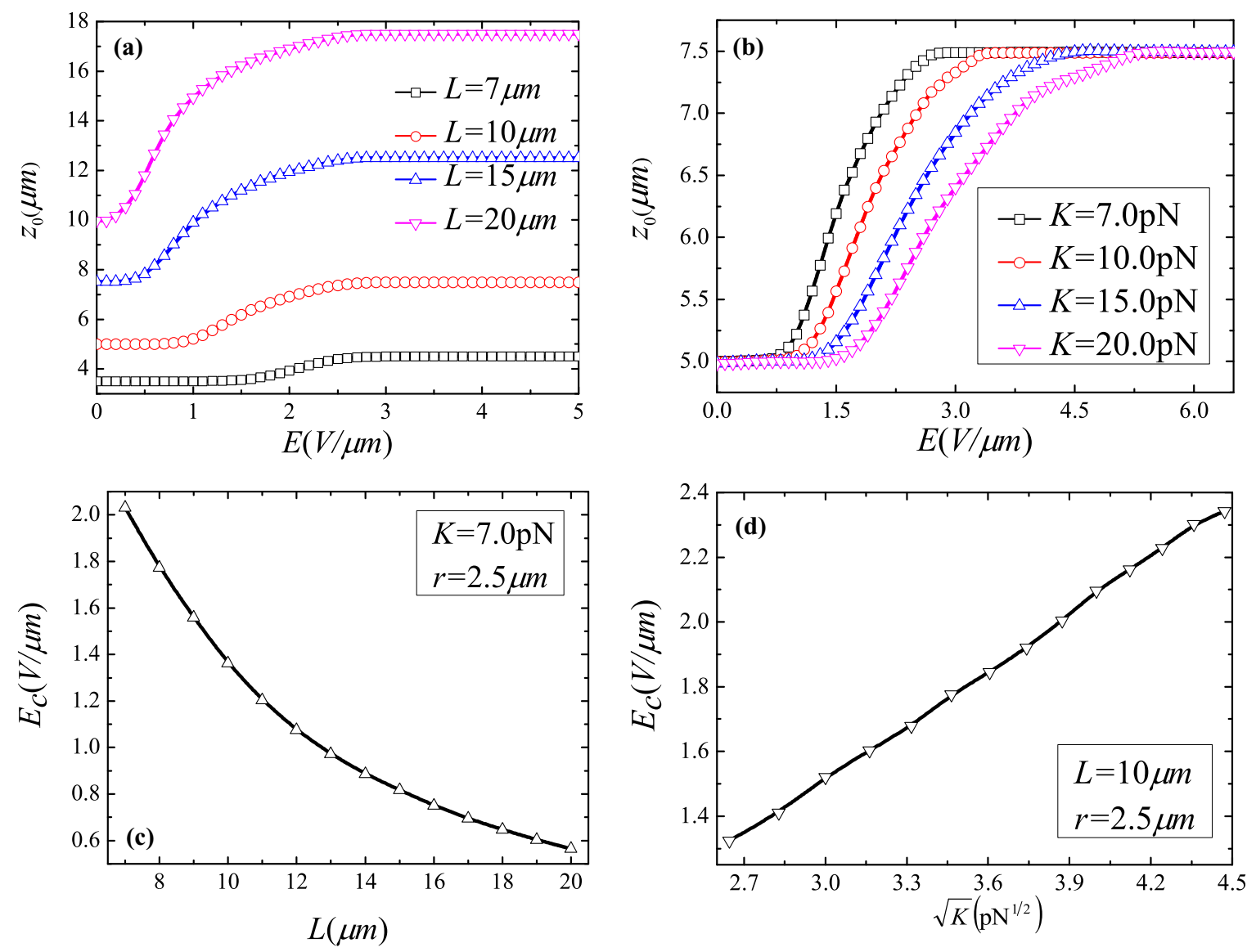

FIG. 3. Equilibrium position $z_{0}$ in response to the electric field for different (a) cell thicknesses (7, 10, 15, and $20 \mu \mathrm{m}$ ), where the Frank elastic constant and the radius of the microdroplet are set as $K=7.0 \mathrm{pN}$ and $r=2.5 \mu \mathrm{m}$ respectively, and (b) Frank elastic constants (7, 10 , 15 , and $20 \mathrm{pN}$ ), where the cell thickness and the radius of the microdroplet are set as $L=10 \mu \mathrm{m}$ and $r=2.5 \mu \mathrm{m}$, respectively. These two figures show a positional transition occurring at an electric field threshold $E_{c}$, which depends on (c) cell thickness $L$ and (d) Frank elastic constant $\sqrt{K}$.

where $r$ is the radius of microdroplet, $p=2.04 r^{2}$ is the magnitudes of the dipole moment, $\rho_{\mathrm{LC}}-\rho_{a q}$ is the density difference between liquid crystal and microdroplet, $g=9.8 \mathrm{~m} / \mathrm{s}^{2}$ is the gravitational acceleration, and $z$ denotes the position of microdroplet. The configuration of the droplet and defect as shown in Fig. 1 corresponds to a positive $p$.

\section{RESULTS AND DISCUSSIONS}

In the presence of a small external field, the total energy given by Eq. (5) overlaps the elastic energy $U_{e}$ and remains symmetric, indicating that the interaction among LC molecules still dominates the system if the external field applied is not large enough to realign the LC molecules, especially in the region close to the midplane. Thus the contribution made by asymmetric gravitational potential is trivial and the microdroplet in this case is still trapped within its midplane, as shown in Figs. 2(b) and 2(c). However, as we increase the field applied, it tends to widen and flatten the bottom of the elastic potential well and that by contrast enlarges the relative contribution made by the asymmetric buoyant force to the total energy. As a result, the buoyant force will drive the microdroplet with ease from midplane to a new equilibrium position [Fig. 2(d)]. It is obvious that the sign of
$\rho_{\mathrm{LC}}-\rho_{a q}$ determines the direction of particle displacement with respect to the location of the singular defect. It seems that the interaction potential well around the midplane tends to be flattened due to the realignment of liquid crystal molecules made by the applied external field, which creates a "fast lane" in the vertical direction for the microdroplet to move. It triggers a positional transition from the midplane, if driven by an asymmetric buoyant force, when such a fast lane is fast enough (weakens the elastic energy gradient).

Furthermore, in order to study the effect of the cell thickness and Frank constant on the critical electric value, we plot the equilibrium position against the applied electric field for different cell thicknesses (7, 10, 15, and $20 \mu \mathrm{m})$ and Frank elastic constants $(7,10,15$, and $20 \mathrm{pN})$, as shown in Figs. 3(a) and 3(b). It is found that a positional transition occurs when the external field applied exceeds a threshold value. The thinner the cell thickness $L$ is and the larger the Frank elastic constant $K$ is, the larger the critical electric field is needed to trigger the transition, as shown in Figs. 3(c) and 3(d). A deeper investigation shows that the critical value of electric field is inversely proportional to $L$ and linearly proportional to $\sqrt{K}$, a Fréedericksz-like behavior.

To gain more insight into the dynamic behaviors of the microdroplet, we plot the threshold value against $\sqrt{K} / L$ to 

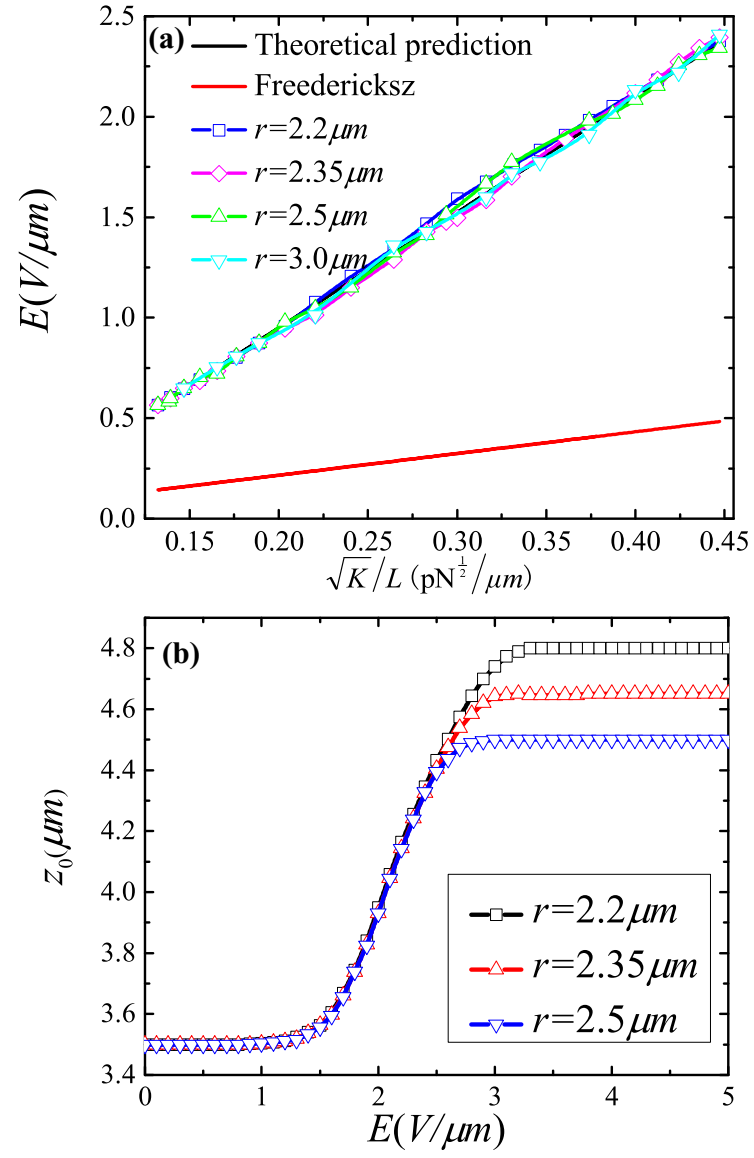

FIG. 4. (a) Dependence of $E_{c}$ and $\sqrt{K} / L$ for different radii of microdroplet $(2.2,2.35,2.5$, and $3.0 \mu \mathrm{m})$, obeying strictly a master curve described by the theoretical prediction (6). (b) Equilibrium position $z_{0}$ for different radii of microdroplet with $K=7 \mathrm{pN}$ and $L=7 \mu \mathrm{m}$, showing the same critical value $E_{c}$ of electric field.

obtain a master curve, as shown in Fig. 4(a), where a Fréedericksz curve (red) is also plotted. It is interesting to find that the critical electric field to trigger a positional transition for a microdroplet suspended in a NLC cell follows a Fréederickszlike linear master curve, yet with a different slope. In addition, one finds no or a slight dependence of the critical value on the size of the liquid droplet, as shown in Fig. 4(b).
Moreover, by comparing the numerical calculation results with the Fréedericksz effect curve $(\pi \sqrt{4 \pi /|\Delta \varepsilon|} \sqrt{K} / L)$ in Fig. 4(a), it is surprising to find that the slope difference between them is by a factor of $3 \sqrt{\pi}$. The additional energy contribution coming from the surface energy due to the introduction of a microdroplet is proportional to $\pi$ (surface area). On the other hand, the energy contribution made by the external field is proportional to $E^{2}$, which gives a critical value of the external field proportional to $\sqrt{\pi}$, because the transition comes from the competition between an equivalent surface energy due to the introduction of microdroplet and the Coulomb interaction due to the application of external field. More specifically, an explicit expression (where $\mathcal{F}$ denotes the Fréedericksz effect)

$$
E_{c}=3 \sqrt{\pi} \mathcal{F}-\frac{1}{5}=6 \pi^{2} \sqrt{\frac{K}{|\Delta \varepsilon| L^{2}}}-\frac{1}{5}
$$

for the critical electric field can be proposed as a theoretical prediction. Such a prediction, as shown by the straight line in Fig. 4(a), agrees very well for different radii of microdroplets $(2.2,2.35,2.5$, and $3.0 \mu \mathrm{m})$. This once again verifies the conclusion that the critical electric field is independent of microdroplet size. The reason might lie in that, in the present theoretical model, the microdroplet is treated as a dipole in a far-field expansion approximation.

\section{CONCLUSION}

In summary, a positional transition is found for a microdroplet suspended in a NLC cell in the presence of an external electric field. The critical value of the electric field that triggers such a transition is independent of droplet size in the far-field approximation and obeys a Fréedericksz-like dependence on the cell thickness and Frank elastic constant in a master law by a factor of $3 \sqrt{\pi}$. Such a theoretical prediction agrees very well with the numerical calculation results.

\section{ACKNOWLEDGMENT}

This work was supported by the National Science Foundation of China under Grants No. 11974292, No. 11574256, and No. 11847318.
[1] B. Comiskey, J. Albert, H. Yoshizawa, and J. Jacobson, Nature (London) 394, 253 (1998).

[2] Z. Wang and J. Zhe, Chip 11, 1280 (2011).

[3] I. I. Smalyukh, Annu. Rev. Condens. Matter Phys. 9, 207 (2018).

[4] Y.-K. Kim, X. Wang, P. Mondkar, E. Bukusoglu, and N. Abbott, Nature (London) 557, 539 (2018).

[5] E. A. Nance, G. F. Woodworth, K. A. Sailor, T.-Y. Shih, Q. Xu, G. Swaminathan, D. Xiang, C. Eberhart, and J. Hanes, Sci. Transl. Med. 4, 149ra119 (2012).
[6] S. J. Woltman, G. D. Jay, and G. P. Crawford, Nat. Mater. 6, 929 (2007).

[7] P. Poulin, V. Cabuil, and D. A. Weitz, Phys. Rev. Lett. 79, 4862 (1997).

[8] M. Vilfan, N. Osterman, M. Čopič, M. Ravnik, S. Žumer, J. Kotar, D. Babič, and I. Poberaj, Phys. Rev. Lett. 101, 237801 (2008).

[9] U. Ognysta, A. Nych, V. Nazarenko, I. Muševič, M. Škarabot, M. Ravnik, S. Žumer, I. Poberaj, and D. Babič, Phys. Rev. Lett. 100, 217803 (2008). 
[10] M. Škarabot, M. Ravnik, S. Žumer, U. Tkalec, I. Poberaj, D. Babič, N. Osterman, and I. Muševič, Phys. Rev. E 77, 031705 (2008).

[11] A. V. Ryzhkova, M. Škarabot, and I. Muševič, Phys. Rev. E 91, 042505 (2015).

[12] C. P. Lapointe, T. G. Mason, and I. I. Smalyukh, Science 326, 1083 (2009).

[13] U. M. Ognysta, A. B. Nych, V. A. Uzunova, V. M. Pergamenschik, V. G. Nazarenko, M. Škarabot, and I. Muševič, Phys. Rev. E 83, 041709 (2011).

[14] S.-J. Kim, B.-K. Lee, and J.-H. Kim, Liq. Cryst. 43, 1589 (2016)

[15] D. Andrienko, M. Tasinkevych, P. Patrício, M. P. Allen, and M. M. Telo da Gama, Phys. Rev. E 68, 051702 (2003).

[16] K. Izaki and Y. Kimura, Phys. Rev. E 87, 062507 (2013).

[17] O. P. Pishnyak, S. Tang, J. R. Kelly, S. V. Shiyanovskii, and O. D. Lavrentovich, Phys. Rev. Lett. 99, 127802 (2007).

[18] O. P. Pishnyak, S. V. Shiyanovskii, and O. D. Lavrentovich, J. Mol. Liq. 164, 132 (2011).

[19] F. L. Calderon, T. Stora, O. Mondain Monval, P. Poulin, and J. Bibette, Phys. Rev. Lett. 72, 2959 (1994).

[20] M. Yada, J. Yamamoto, and H. Yokoyama, Phys. Rev. Lett. 92, 185501 (2004).

[21] K. Takahashi, M. Ichikawa, and Y. Kimura, Phys. Rev. E 77, 020703(R) (2008).

[22] M. Škarabot, A. V. Ryzhkova, and I. Muševič, J. Mol. Liq. 267, 384 (2018).
[23] H. Stark, Phys. Rep. 351, 387 (2001).

[24] H. Stark, Phys. Rev. E 66, 032701 (2002).

[25] Y. Wang, P. Zhang, and J. Z. Y. Chen, Phys. Rev. E 96, 042702 (2017).

[26] X. Yao, H. Zhang, and J. Z. Y. Chen, Phys. Rev. E 97, 052707 (2018).

[27] P. Poulin, H. Stark, T. C. Lubensky, and D. A. Weitz, Science 275, 1770 (1997).

[28] P. Poulin and D. A. Weitz, Phys. Rev. E 57, 626 (1998).

[29] T. C. Lubensky, D. Pettey, N. Currier, and H. Stark, Phys. Rev. E 57, 610 (1998).

[30] B. Senyuk, O. Puls, O. M. Tovkach, S. B. Chernyshuk, and I. I. Smalyukh, Nat. Commun. 7, 10659 (2016).

[31] R. W. Ruhwandl and E. M. Terentjev, Phys. Rev. E 54, 5204 (1996).

[32] R. W. Ruhwandl and E. M. Terentjev, Phys. Rev. E 56, 5561 (1997).

[33] J. C. Loudet and P. Poulin, Phys. Rev. Lett. 87, 165503 (2001).

[34] S. B. Chernyshuk and B. I. Lev, Phys. Rev. E 81, 041701 (2010).

[35] S. B. Chernyshuk and B. I. Lev, Phys. Rev. E 84, 011707 (2011).

[36] S. B. Chernyshuk, O. M. Tovkach, and B. I. Lev, Phys. Rev. E 85, 011706 (2012).

[37] S.-J. Kim and J.-H. Kim, Soft Matter 10, 2664 (2014).

[38] B.-K. Lee, S.-J. Kim, B. Lev, and J.-H. Kim, Phys. Rev. E 95, 012709 (2017). 